\section{Bundesregierung will Prävention fördern}

- Mit einer nationalen Präventionsstrategie wollen CDU/CSU und FDP erreichen, dass der Deutsche Bundestag konkrete Präventionsziele für die Zeit 2012-2020 beschließt. Er soll sich dabei an Empfehlungen orientieren, die von Expertengremien erarbeitet wurden. Durch gesetzliche Regelungen und durch koordinierende Maßnahmen will man parallel darauf hinwirken, dass alle Präventionsträger ihre Anstrengungen auf diese Ziele konzentrieren. Die Bundesregierung soll deshalb die Zielerreichungsgrade dokumentieren und dem Deutschen Bundestag im Abstand von zwei Jahren über die Ergebnisse berichten.

Um den bereichsübergreifenden Ansatz von Prävention und Gesundheitsförderung weiter voranzubringen, sollen die gesetzlichen Krankenkassen und andere Präven- tionsträger zur Ko-Finanzierung verpflichtet werden. Dazu wird der in $\$ 20$ Absatz 2 $S G B V$ vorgesehene Aufwand, den Kassen pro Versichertem für Präventionsmaßnahmen einsetzen dürfen, auf 6 Euro angehoben. Damit die Kassen hier auch aktiv werden, sollen nicht verausgabte Mittel vom Spitzenverband Bund thesauriert und für Modellvorhaben und Präventionskampagnen eingesetzt werden. Die PKV soll für ihren Verantwortungsbereich finanzielle Mittel in vergleichbarem Umfang für betriebliche und außerbetriebliche SettingMaßnahmen zur Verfügung stellen.

\section{MMW Kommentar}

Nach Auffassung der Unionspolitiker können insbesondere Kur- und Reha-Maßnahmen einen erheblichen Beitrag zur Prävention leisten. Deshalb sollen Anreize geschaffen wer- den, damit solche Maßnahmen verstärkt in Anspruch genommen werden. Schließlich könnten qualitätsgesicherte und effiziente Reha-Maßnahmen Kosten in anderen Bereichen vermeiden helfen. Kurmaßnahmen als präventivmedizinische Leistungen könnten dazu beitragen, Krankheiten zu verhindern und Lebenskraft zu erhalten.

Dies treffe auch aufpflegende Angehörige $z u$, die in ihrer besonderen Situation extremen Belastungen ausgesetzt seien. Deshalb soll auch im Rahmen der anstehenden Reform der Pflegeversicherung ein Anspruch von pflegenden Angehörigen auf medizinisch indizierte Kurmaßnahmen geschaffen werden. Der Anspruch von Müttern und Vätern auf Vorsorge- und Reha-Leistungen sei zwar gesetzlich kontinuierlich verbessert worden. Die Krankenkassen wären aber in der Pflicht, die gesetzlichen Vorgaben umzusetzen und ihre Genehmigungspraxis zu verbessern. Für die Praxis könnte man diese Argumente schon jetzt verwenden, wenn eine solche Reha-Maßnahme bei einem Patienten wieder einmal abgelehnt wurde.

rechnungsbelege, aber auch die Überweisungen nach Muster 10 beziehungsweise 10a übertragen werden. Treffen bei einem Patienten mehrere ATB zu, reicht eine Angabe aus, damit der Behandlungsfall nicht in das Laborbudget fällt, beziehungsweise die so gekennzeichneten Laborleistungen nicht gegen das Budget gerechnet werden. Wenn das Praxisverwaltungssystem umgestellt wird, neue Updates aufgespielt wurden oder das Labor bei der Übermittlung der Daten Umstellungen vornimmt, lohnt es sich zu überprüfen, ob die ATB korrekt übertragen wurden. Es lohnt sich auch, den Abrechnungsbescheid in dieser Richtung gezielt zu überprüfen, denn erst nach dem bundesweiten Laborclearing kann sicher festgestellt werden, ob die auf den Laboraufträgen angegebenen ATB auch tatsächlich korrekt berücksichtigt wurden. 